\title{
Eight-hours adaptive deep brain stimulation in patients with Parkinson disease
}

Mattia Arlotti, PhD,* Sara Marceglia, PhD,* Guglielmo Foffani, PhD, Jens Volkmann, MD, PhD, Andres M. Lozano, MD, PhD, Elena Moro, MD, PhD, Filippo Cogiamanian, MD, Marco Prenassi, Ms, Tommaso Bocci, MD, Francesca Cortese, MD, Paolo Rampini, MD, Sergio Barbieri, MD, PhD, and Alberto Priori, MD, PhD

Neurolog $y^{\circledR}$ 2018;90:e971-e976. doi:10.1212/WNL.0000000000005121

\section{Abstract}

\section{Objectives}

To assess the feasibility and clinical efficacy of local field potentials (LFPs)-based adaptive deep brain stimulation (aDBS) in patients with advanced Parkinson disease (PD) during daily activities in an open-label, nonblinded study.

\section{Methods}

We monitored neurophysiologic and clinical fluctuations during 2 perioperative experimental sessions lasting for up to 8 hours. On the first day, the patient took his/her daily medication, while on the second, he/she additionally underwent subthalamic nucleus aDBS driven by LFPs beta band power.

\section{Results}

The beta band power correlated in both experimental sessions with the patient's clinical state (Pearson correlation coefficient $r=0.506, p<0.001$, and $r=0.477, p<0.001$ ). aDBS after LFP changes was effective (30\% improvement without medication [3-way analysis of variance, interaction day $\times$ medication $p=0.036 ; 30.5 \pm 3.4$ vs $22.2 \pm 3.3, p=0.003]$ ), safe, and well tolerated in patients performing regular daily activities and taking additional dopaminergic medication. aDBS was able to decrease DBS amplitude during motor "on" states compared to "off" states (paired $t$ test $p=0.046$ ), and this automatic adjustment of STN-DBS prevented dyskinesias.

\section{Conclusions}

The main findings of our study are that aDBS is technically feasible in everyday life and provides a safe, well-tolerated, and effective treatment method for the management of clinical fluctuations.

\section{Classification of evidence}

This study provides Class IV evidence that for patients with advanced PD, aDBS is safe, well tolerated, and effective in controlling PD motor symptoms.

\author{
Correspondence \\ Prof. Priori \\ alberto.priori@unimi.it
}

\section{MORE ONLINE}

$\rightarrow$ Class of Evidence NPub.org/coe

\section{RELATED ARTICLE}

\section{Editorial}

Approaching adaptive control in neurostimulation for Parkinson disease: Autopilot on

Page 497 


\section{Glossary}

aDBS = adaptive deep brain stimulation; AE = adverse event; ANOVA = analysis of variance; $\mathbf{D B S}=$ deep brain stimulation; LFP = local field potential; PD = Parkinson disease; UDysRS = Unified Dyskinesias Rating Scale; UPDRS III = Unified Parkinson's Disease Rating Scale, part III.

Optimizing deep brain stimulation (DBS) therapy for patients with Parkinson disease (PD) now seems feasible thanks to the successful results of preliminary studies on closed-loop, adaptive DBS (aDBS) strategies aimed at providing the best stimulation parameters according to the patient's clinical state. $^{1-5}$ aDBS is thought to overcome classic DBS therapy limitations, including stimulation-induced side effects in the long term such as gait and speech impairment and the presence of motor fluctuations not well controlled. ${ }^{6-8}$

To adapt stimulation parameters, aDBS uses the synchronous presynaptic and postsynaptic activity of local neuronal populations (local field potentials [LFPs]) recorded through the same implanted electrodes delivering stimulation. The power of the beta band oscillation $(11-35 \mathrm{~Hz})$, which is modulated by levodopa administration, movement execution and preparation, motor planning, and DBS, ${ }^{6-8}$ is considered the most promising biomarker for aDBS.

Two main adaptation strategies have been tested in humans. The first one is based on an on-off approach that activates DBS only when the LFP beta power exceeds a personalized threshold, ${ }^{1,2}$ and the second approach linearly modulates the stimulation voltage according to the changes in the LFP beta power, adapting moment-by-moment DBS parameters to the patient's state. ${ }^{3,4}$ In both cases, aDBS proved to be more effective than conventional DBS in improving motor scores, ${ }^{1}$ in controlling levodopa-induced dyskinesias, ${ }^{3,4}$ and in reducing speech-related side effects. ${ }^{5}$

Despite the positive results, available data were obtained in short experimental sessions, up to 2 hours. ${ }^{3,4}$

As a preliminary step for developing an implantable device, in this work, we aimed to investigate in a long time window in an open-label, nonblinded study whether aDBS is able to follow and control clinical fluctuations using beta band power changes and is well tolerated by and safe for patients.

\section{Methods}

We enrolled 13 rigid-akinetic patients with advanced PD who underwent surgery for subthalamic nucleus DBS electrode implantation in the Neurosurgery Unit at Fondazione IRCCS Ca'Granda Ospedale Maggiore Policlinico in Milan from March 2016 to January 2017 without experiencing any surgical complication. The study was approved by the institutional review board and conformed with the Declaration of Helsinki, and all patients provided written informed consent to the experimental procedures. Patients were implanted bilaterally but studied unilaterally. The choice of the side is detailed in appendix e-1, http://links.lww.com/ WNL/A238.

The experimental sessions took place after DBS surgery before the leads were connected to the subcutaneous pulse generator.

Before the experimental session started, a calibration session was performed to verify the presence of a significant beta peak in at least 1 side, to establish the best side for recording LFPs and testing aDBS, and to establish effective stimulation parameters (appendix e-1, http://links.lww.com/WNL/ A238). Because, according to the literature, ${ }^{9}$ we expected that the beta peak was not detectable in all patients and because the current aDBS algorithm is based on beta band modulations, those not showing a beta peak were excluded from the study.

The experimental protocol (figure 1A) included two 8-hour sessions ( 1 per day) conducted with a portable aDBS prototype device? : day 1 , stimulation "off" and LFP recordings "on"; and day 2, stimulation "on" (aDBS) and LFP recordings "on."

After 12 hours of medication withdrawal, each session started with a baseline assessment (medication "off," stimulation "off"). The patient took his/her first morning medication afterward and was then assessed when his/her medication was effective (medication "on," peak dose $\approx 45-60$ minutes after medication intake) and then when the medication ended its effects (medication "off," end dose 60-90 minutes after the peak dose). The same procedure was repeated throughout the 8 hours (figure 1A). According to the personal response timing, each patient took 2 or 3 medications. Hence, to allow comparisons, we considered the clinical assessments related to the first 2 administrations. On day 2, DBS was switched "on" at the beginning of the experimental session, immediately after the baseline assessment. The clinical assessments on day 2 followed the same schema as on day 1 , according to levodopa response (peak dose, end dose).

For the clinical assessment, we collected the Unified Parkinson's Disease Rating Scale, part III, motor part (UPDRS III), the Unified Dyskinesias Rating Scale (UDysRS), parts 3 and 4, and the adverse events (AEs) experienced by the patient (appendix e-1, http://links.lww. com/WNL/A238 for AE classification). 

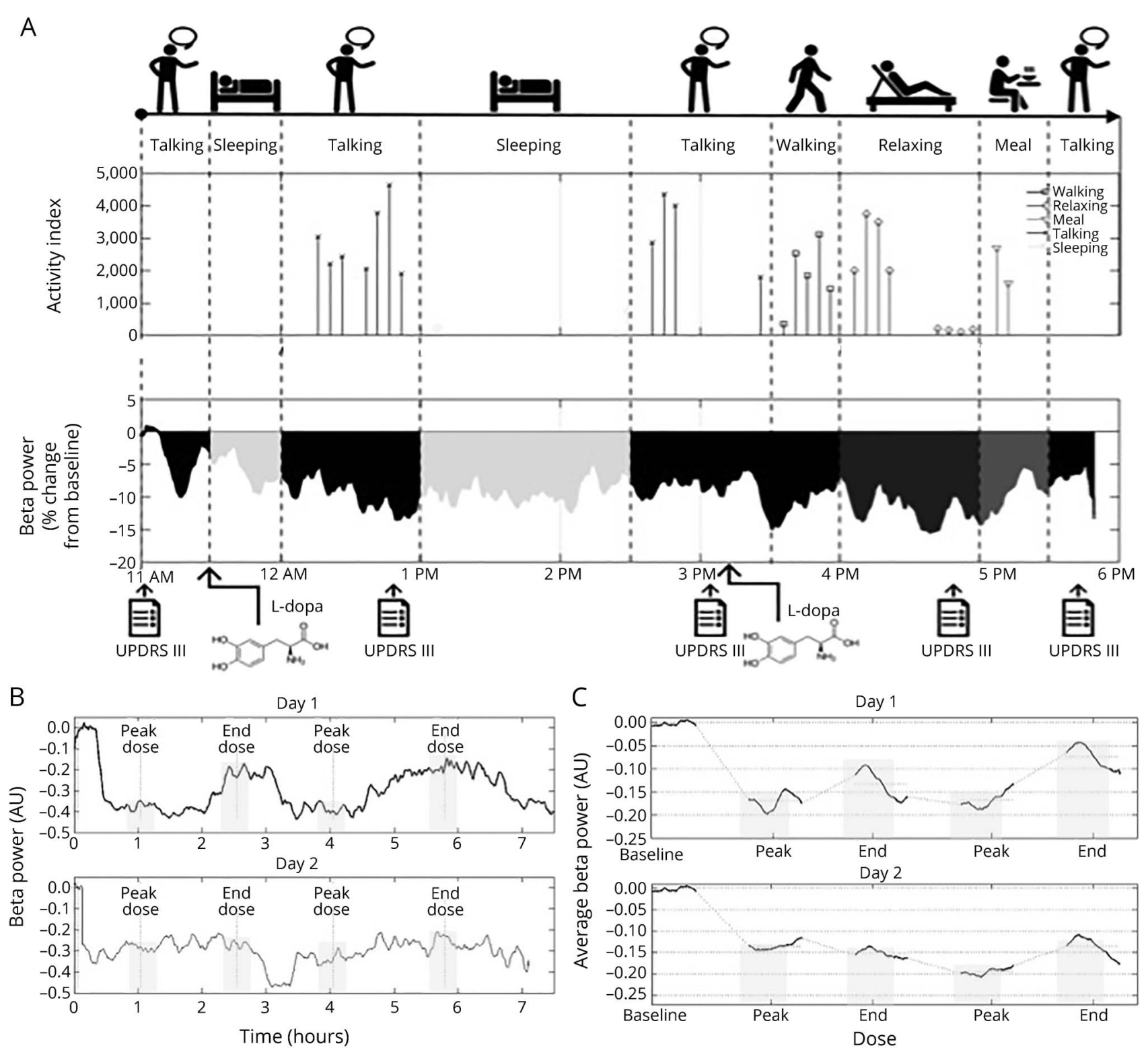

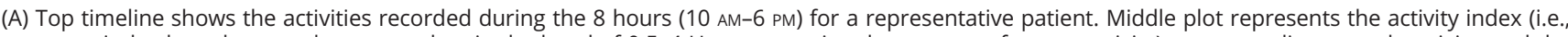

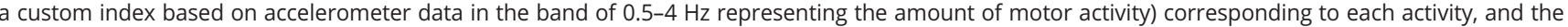
bottom trace is beta power percentage change from baseline throughout the 8 hours. Bottom arrows show the times in which the patient was assessed

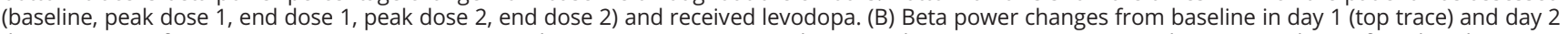

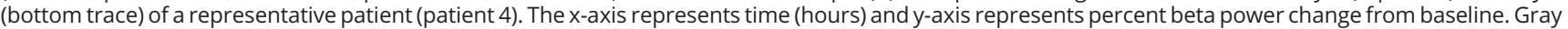
rectangles highlight beta power during clinical assessments (baseline, peak dose 1, end dose 1, peak dose 2, end dose 2). (C) Average beta power changes

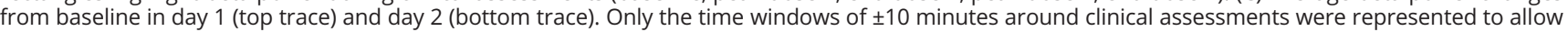

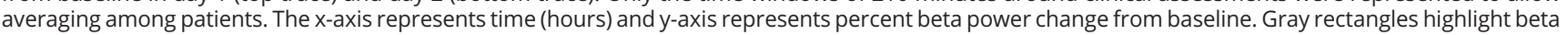
power during clinical assessments (baseline, peak dose 1, end dose 1, peak dose 2, end dose 2). aDBS = adaptive deep brain stimulation; AU = arbitrary units;

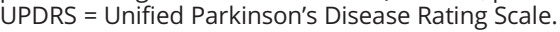

To assess the aDBS device function throughout the whole 8 -hour session, we collected the beta band power and, on day 2 , the stimulation amplitude. We correlated the clinical assessment with the beta power corresponding to the time at which the UPDRS III was assessed ( \pm 10 minutes). We also collected the patient's physical activity using a wearable commercial bracelet equipped with a 3-axis accelerometer. A dedicated application was developed to acquire and store data and to provide a clinical diary (sleep, rest, walk, talk, eat, other) to be filled in by the patient every 30 minutes.

We used data on day 1 to verify the ability of beta band power to act as a biomarker of patient's state, and more specifically, we tested whether the beta power was modulated by levodopa using nonsupraliminar levodopa doses (paired $t$ test beta power "off" vs beta power "on," $p<0.05)$, the beta power 
correlated to the patient's clinical state as measured by the UPDRS III (Pearson correlation coefficient, $p<0.05$ ), and the beta power correlated with the specific physical activities as measured by the wearable bracelet (Pearson correlation coefficient, $p<0.05$ ).

aDBS efficacy was assessed by a comparison of clinical data (UPDRS III and UDysRS) collected on day 1 vs 2 (3-way analysis of variance [ANOVA] with the factors day [2 levels, day 1 and day 2], condition [2 levels, medication "on" and medication "off"], and administration [2 levels, administration 1 and administration 2]). aDBS safety was verified by the occurrence and intensity of AEs related to stimulation. Data here are reported as mean \pm standard error.

\section{Results}

We screened 13 patients (table 1). Of them, only 1 patient (patient 5) did not show a significant beta peak, and we lost the entire day 1 recording of patient 6 because of battery discharge. Hence, we analyzed 11 patients. Experimental details are reported in table e-1, http://links.lww.com/WNL/A237.

In day 1, we found that the beta power responded to levodopa administration, as expected, with an average decrease of $18.0 \pm$ $0.03 \%$ in peak dose compared to a $8.3 \pm 0.03 \%$ in end dose ( $p=0.009$ ) with respect to baseline (medication "off," stimulation "off," after 12 hours of levodopa withdrawal). In addition, the beta power was confirmed to correlate with the patient's clinical state as measured by the UPDRS III (Pearson correlation coefficient $=0.477, p<0.001$ ) and was specifically modulated during walking with respect to talking and relaxing (beta power change from baseline during: walking $-14.0 \pm$ $4.212 \%$, talking $-11.2 \pm 2.724 \%$, relaxing $-8.8 \pm 2.418 \%$, 1 way ANOVA $p<0.0001$ ).

On day 2 (figure 2), we observed that aDBS was effective in reducing UPDRS III motor scores by almost $30 \%$ when the patients were "off" medication, whereas no improvement was observed in day 1 when the patients did not receive aDBS (baseline vs medication "off": day $1,32.6 \pm 2.8$ vs $29.4 \pm 3.9$; day 2, $30.5 \pm 3.4$ vs $22.2 \pm 3.3$; 3-way ANOVA interaction day $\times$ levodopa $p=0.036$; post hoc test $p=0.003$ ). When the patients were "on" levodopa, the cumulative effect of aDBS and levodopa provided a $45 \%$ improvement in the UPDRS III motor scores that was comparable to that elicited by levodopa alone on day 1 (baseline vs medication "on": day $1,32.6 \pm 2.8$ vs $17.9 \pm 2.0$; day $2,30.5 \pm 3.4$ vs $15.5 \pm 2.3$; post hoc test $p=$ $0.4)$. The cumulative effect of levodopa and aDBS did not elicit dyskinesias (medication "off" vs medication "on": day 1 , $3.4 \pm 1.0$ vs $5.8 \pm 1.7$; day 2 , $3.5 \pm 0.9$ vs $4.4 \pm 1.5$; 3-way ANOVA interaction day $\times$ levodopa $p=0.51$ ). In fact, as expected, the amount of stimulation voltage delivered when the patient was on levodopa peak was significantly lower than that delivered when the patient was in the end peak

Table 1 Patients' preoperative clinical details

\begin{tabular}{|c|c|c|c|c|c|c|c|c|c|}
\hline & \multirow[b]{2}{*}{ Case } & \multirow[b]{2}{*}{ Sex } & \multirow[b]{2}{*}{ Age, $y$} & \multirow[b]{2}{*}{$\begin{array}{l}\text { Disease } \\
\text { duration, y }\end{array}$} & \multirow[b]{2}{*}{$\begin{array}{l}\text { Fluctuation } \\
\text { onset, y }\end{array}$} & \multirow[b]{2}{*}{$\begin{array}{l}\text { Onset } \\
\text { side }\end{array}$} & \multirow[b]{2}{*}{$\begin{array}{l}\text { Preoperative } \\
\text { LEDD }\end{array}$} & \multicolumn{2}{|c|}{ Preoperative response to levodopa ${ }^{a}$} \\
\hline & & & & & & & & $\begin{array}{l}\text { UPDRS III } \\
\text { score, } \\
\text { medication } \\
\text { "off" }\end{array}$ & $\begin{array}{l}\text { UPDRS III score, } \\
\text { medication "on" }\end{array}$ \\
\hline 1 & 1 & M & 59 & 10 & 6 & $\mathrm{R}$ & 208 & 25 & 13 \\
\hline 2 & 2 & M & 62 & 9 & 6 & $\mathrm{R}$ & 685.55 & 41 & 18 \\
\hline 3 & 3 & M & 67 & 12 & 5 & $\mathrm{R}$ & 1,494 & 25 & 10 \\
\hline 4 & 4 & M & 50 & 8 & 5 & $\mathrm{R}$ & 1,055 & 32 & 16 \\
\hline 5 & 5 & M & 47 & 11 & 2.5 & $\mathrm{R}$ & 1,080 & 19 & 10 \\
\hline 6 & 6 & M & 58 & 7 & 2 & $\mathrm{R}$ & $1,856.66$ & 37 & 11 \\
\hline 7 & 7 & $\mathrm{~F}$ & 65 & 14 & 4 & L & 652 & 22 & 8 \\
\hline 8 & 8 & M & 70 & 12 & 2 & L & 872 & 13 & 3 \\
\hline 9 & 9 & $\mathrm{~F}$ & 47 & 7 & 2 & L & $1,436.1$ & 24 & 8 \\
\hline 10 & 10 & M & 54 & 13 & 4.5 & $\mathrm{~L}$ & 785 & 24 & 8 \\
\hline 11 & 11 & M & 59 & 14 & 12 & L & 665 & 30 & 10 \\
\hline 12 & 12 & $\mathrm{~F}$ & 58 & 11 & 5 & L & 1,250 & 39 & 16 \\
\hline 13 & 13 & $\mathrm{~F}$ & 70 & 13 & 7 & L & 208 & 25 & 12 \\
\hline
\end{tabular}



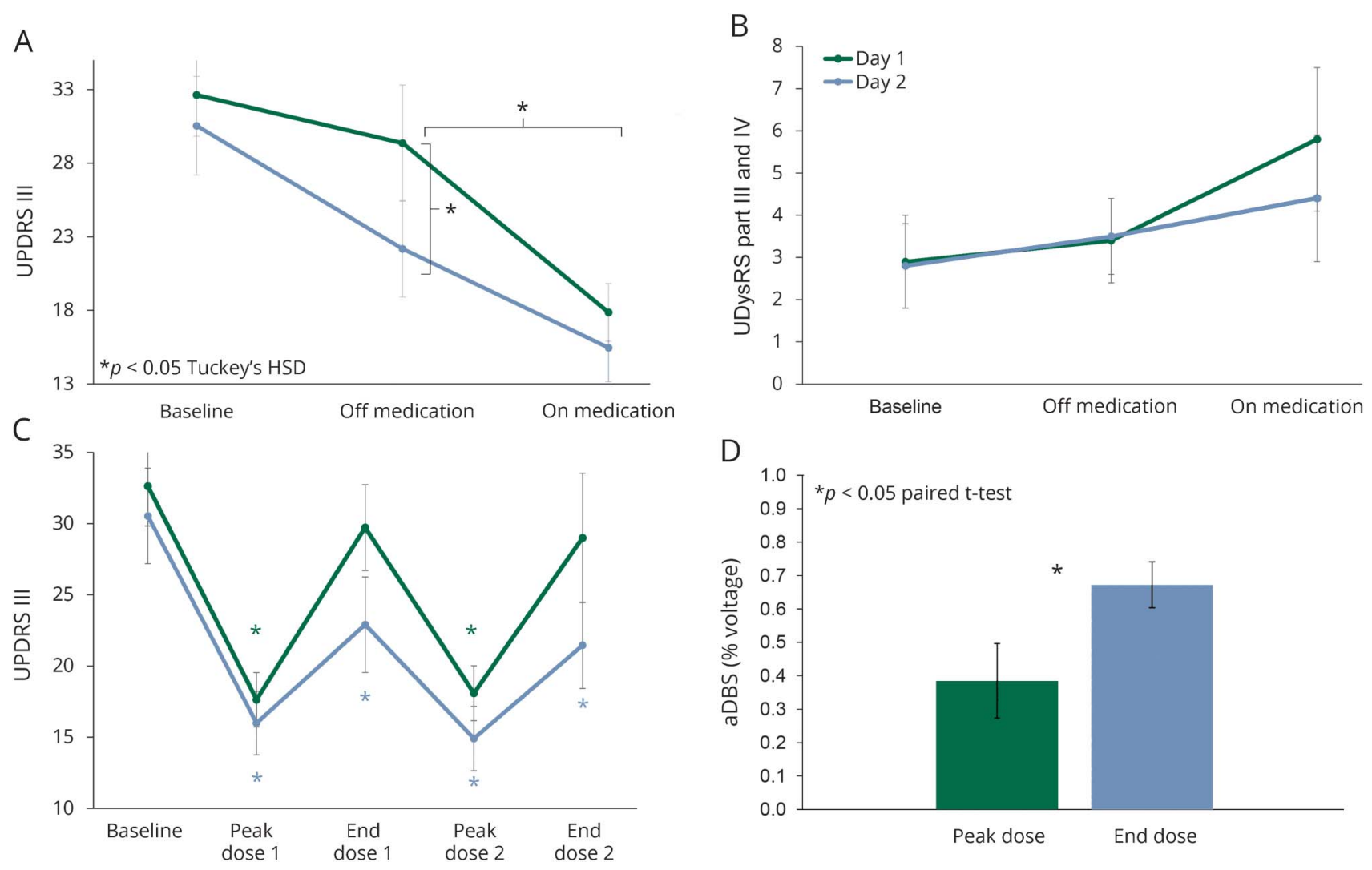

(A) Graph represents average UPDRS III values on day 1 (green line) and day 2 (light gray line) at baseline, at end dose/medication "off," and at peak dose/ medication "on." Error bars are SEMs. UPDRS III values at end dose/medication "off" on day 1 were significantly different from those on day 2 and from those on peak dose/medication "on" on the same day 1. (B) Graph represents average UDysRS, part III and IV values on day 1 (green line) and on day 2 (light gray line) at baseline, at end dose/medication "off," and at peak dose/medication "on." Error bars are SEMs. No significant differences were detected among conditions. (C) Details of the UPDRS III values on day 1 (green line) and on day 2 (light gray line) at baseline, at end dose/medication "off," and at peak dose/medication "on" in the 2 sessions throughout the whole 8-hour experiment. On day 1, only values at peak dose/medication "on" were different from baseline values, whereas on day 2 , the difference was significant at all time points after aDBS was turned on. (D) Histogram represents the average voltage change in the peak dose/ medication "on" condition (green) and in the end dose/medication "off" (light gray) during day 2. Error bars are SEMs. aDBS = adaptive deep brain stimulation; HSD = highest significant difference; UPDRS = Unified Parkinson's Disease Rating Scale; UDysRS = Unified Dyskinesias Rating Scale.

(normalized stimulation voltage as a percent of the maximum voltage in table e-1, http://links.lww.com/WNL/A237, medication "off" vs medication "on": $0.67 \pm 0.23$ vs $0.38 \pm$ 0.37 , paired $t$ test $p=0.046$ ). As a further verification of the aDBS functioning, we found that the beta power was confirmed to correlate with the patient's clinical state as measured by the UPDRS III (Pearson correlation coefficient $=0.506$, $p<0.001)$ even in day 2. Finally, no AEs specifically related to aDBS or complaints regarding either the experimental procedure or the device/stimulation were reported.

\section{Discussion}

In our 8-hour experiment, our aDBS device provided a stimulation output that was always calibrated to the beta rhythm and to the patient's state.

To the best of our knowledge, this is the longest experiment with aDBS. Previous experiments with aDBS from our group and other laboratories showed aDBS effectiveness in shorter time windows. ${ }^{1-5}$ Our observations showed that changing DBS voltage linearly with beta rhythm provides constant benefit for hours of unrestricted patient activity and in conjunction with normal levodopa assumption. Conversely, in previous experiments, to understand whether aDBS was able to control stimulation side effects better than conventional DBS, patients were administered a supraliminal dose of levodopa, able to induce dyskinesias, especially if summed to DBS.,

Despite these new insights provided by the current experiment, this study has some limitations. First, aDBS was delivered with an external prototype immediately after the surgery for DBS electrode placement, in the so-called acute experimental setting. In this time window, several adaptation processes at the level of the tissue-electrode interface occur, and the presence of edema and the lesional effect due to electrode insertion limit the possibility to mimic the more stable chronic phase. In addition, unilateral aDBS was applied, and the study was conducted in an open-label, unblinded fashion. Finally, the analysis of daily of life activities (e.g., talking, relaxing, sleeping) was based on a self-reporting diary 
and on the data from a single accelerometer placed on the patient's wrist in 30-minute time windows. This provided an indication of the predominance of a certain activity during this time frame, and it cannot track the probable co-occurrence of different activities. To account for this intrinsic inaccuracy, the beta power was studied in average during the time frame. This approach, however, provided evidence of an average correlation between the beta power and the patient's activity that should be better studied with more quantitative approaches based on multiple sensors.

Although still obtained in an acute experimental setting a few days after DBS surgery, our results show that beta band-based aDBS can induce stable control of PD-related motor disturbances by adapting stimulation parameters according to the patient's state. Overall, this finding is a step forward in the development of the new generation of implantable aDBS devices for treating PD.

\section{Author contributions}

Mattia Arlotti: study design and concept, data acquisition, analysis and interpretation, manuscript drafting. Sara Marceglia: study concept, data analysis and interpretation, statistical analysis, manuscript drafting. Guglielmo Foffani: data interpretation, critical revision of manuscript for intellectual content. Jens Volkmann, Andres M. Lozano, and Elena Moro: critical revision of manuscript for intellectual content. Filippo Cogiamanian: data acquisition. Marco Prenassi: data analysis. Tommaso Bocci, Francesca Cortese, and Paolo Rampini, data acquisition. Sergio Barbieri: critical revision of manuscript for intellectual content. Alberto Priori: study concept and design, critical revision of manuscript for intellectual content.

\section{Study funding}

The study was partly supported by donation in memory of Aldo Ravelli and by grant GR-2011-02352807 from the Italian Ministry of Health.

\section{Disclosure}

M. Arlotti reports no disclosures relevant to the manuscript. S. Marceglia and G. Foffani: shareholder of Newronika Srl, a spinoff company of the Fondazione IRCCS Ca'Granda Ospedale Maggiore Policlinico and of the University of Milan. J. Volkmann, A. Lozano, and E. Moro: member of the scientific advisory board of Newronika Srl, a spinoff company of the Fondazione IRCCS Ca'Granda Ospedale Maggiore Policlinico and of the University of Milan. F. Cogiamanian: shareholder of Newronika Srl, a spinoff company of the Fondazione IRCCS Ca'Granda Ospedale Maggiore Policlinico and of the University of Milan. M. Prenassi, T. Bocci, and F. Cortese report no disclosures relevant to the manuscript. P. Rampini, S. Barbieri, and A. Priori: shareholder of Newronika Srl, a spinoff company of the Fondazione IRCCS Ca'Granda Ospedale Maggiore Policlinico and of the University of Milan. Go to Neurology.org/N for full disclosures.

Received August 4, 2017. Accepted in final form December 20, 2017.

\section{References}

1. Little S, Pogosyan A, Neal S, et al. Adaptive deep brain stimulation in advanced Parkinson disease. Ann Neurol 2013;74:449-457.

2. Little $S$, Beudel M, Zrinzo L, et al. Bilateral adaptive deep brain stimulation is effective in Parkinson's disease. J Neurol Neurosurg Psychiatry 2016;87:717-721.

3. Rosa M, Arlotti M, Ardolino G, et al. Adaptive deep brain stimulation in a freely moving Parkinsonian patient. Mov Disord 2015;30:1003-1005.

4. Rosa M, Arlotti M, Marceglia S, et al. Adaptive deep brain stimulation controls levodopa-induced side effects in Parkinsonian patients. Mov Disord Epub 2017 Feb 17.

5. Little S, Tripoliti E, Beudel M, et al. Adaptive deep brain stimulation for Parkinson's disease demonstrates reduced speech side effects compared to conventional stimulation in the acute setting. J Neurol Neurosurg Psychiatry Epub 2016 Aug 16.

6. Fasano A, Aquino CC, Krauss JK, Honey CR, Bloem BR. Axial disability and deep brain stimulation in patients with Parkinson disease. Nat Rev Neurol 2015;11:98-110.

7. Rodriguez-Oroz MC, Moro E, Krack P. Long-term outcomes of surgical therapies for Parkinson's disease. Mov Disord 2012;27:1718-1728.

8. Krack P, Martinez-Fernandez R, del Alamo M, Obeso JA. Current applications and limitations of surgical treatments for movement disorders: surgical treatments for movement disorders. Mov Disord 2017;32:36-52.

9. Giannicola G, Marceglia S, Rossi L, et al. The effects of levodopa and ongoing deep brain stimulation on subthalamic beta oscillations in Parkinson's disease. Exp Neurol 2010;226:120-127. 


\section{Eight-hours adaptive deep brain stimulation in patients with Parkinson disease}

Mattia Arlotti, PhD, Sara Marceglia, PhD, Guglielmo Foffani, PhD, Jens Volkmann, MD, PhD, Andres M. Lozano, MD, PhD, Elena Moro, MD, PhD, Filippo Cogiamanian, MD, Marco Prenassi, Tommaso Bocci, MD, Francesca Cortese, MD, Paolo Rampini, MD, Sergio Barbieri, MD, PhD, and Alberto Priori, MD, PhD

Correspondence Dr. Priori alberto.priori@unimi.it

Cite as: Neurology ${ }^{\circledR}$ 2018;90:e971-e976. doi:10.1212/WNL.0000000000005121

\section{Study question}

Is local field potential (LFP)-based adaptive deep brain stimulation (aDBS) administered during daily activities feasible and effective for patients with advanced Parkinson disease (PD)?

\section{Summary answer}

LFP-based aDBS is a feasible, safe, well-tolerated, and effective way to manage these patients' clinical fluctuations.

\section{What is known and what this paper adds}

LFP-based aDBS may provide improvements over classic DBS techniques, but available evidence comes from experimental sessions that only lasted up to 2 hours. This study provides Class IV evidence for the feasibility and efficacy of LFP-based aDBS over longer sessions, up to 8 hours.

\section{Participants and setting}

This study examined 13 patients with rigid-akinetic advanced PD at a Department of Clinical Neurology and Neurosurgery in Milan, Italy between March 2016 and January 2017.

\section{Design, size, and duration}

The participants underwent surgical insertion of DBS electrodes into the subthalamic nucleus and then underwent two 8-hour experimental sessions over consecutive days. LFP recordings were conducted during both sessions, but aDBS was activated only in the second session. The patients took their prescribed medications on both days. Clinical assessments were made based on the Unified PD Rating Scale (UPDRS), the Unified Dyskinesias Rating Scale, and adverse events.

\section{Primary outcomes}

The primary outcomes were correlations during long lasting recordings (up to 8 hours) between beta band power and UPDRS scores and comparisons between UPDRS scores on days 1 and 2 .

\section{Main results and the role of chance}

Beta band power correlated with UPDRS scores on days 1 (Pearson's $r=0.477 ; p<0.001$ ) and 2 (Pearson's $r=0.506 ; p$ $<0.001)$. Activating aDBS achieved a nearly $30 \%$ reduction in

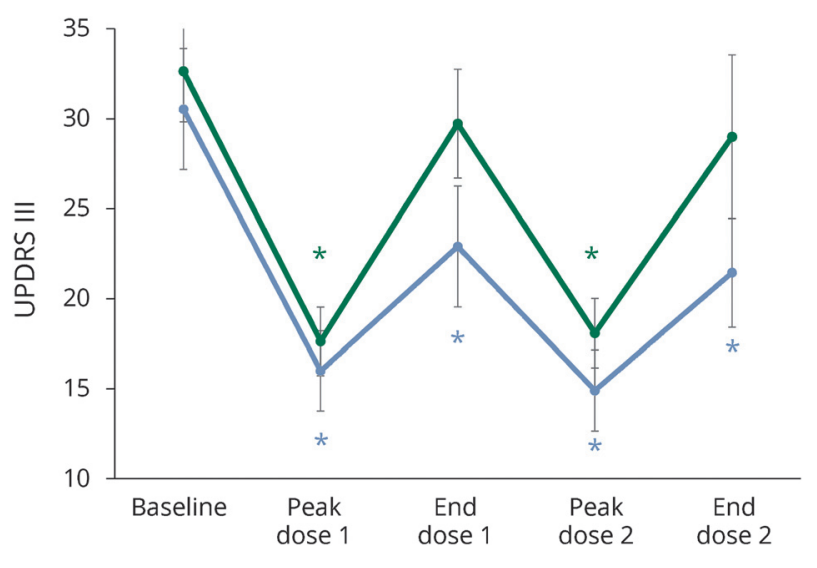

day 2 UPDRS motor scores when patients were "off" levodopa that was not observed when comparing analogous timepoints on day $1(p=0.003)$. When patients were "on" levodopa, the combined effect of aDBS and levodopa on day 2 was a $\sim 45 \%$ reduction in UPDRS motor scores comparable to that observed for levodopa alone on day $1(p=0.4)$. No aDBS-related adverse events, including dyskinesia, were reported.

\section{Bias, confounding, and other reasons for caution}

This was an unblinded open-label study. The experiments were conducted immediately after implantation surgeries, so adaptation processes had not yet occurred.

\section{Generalizability to other populations}

The aDBS algorithm here validated is specific for PD-STN target, but the feasibility and safety of the aDBS approach is potentially generalizable to other DBS indications.

\section{Study funding/potential competing interests}

This study was funded by private donations and the Italian government. Some authors report having financial interests in Newronika Srl. Go to Neurology.org/N for full disclosures. 


\section{Neurology}

\section{Eight-hours adaptive deep brain stimulation in patients with Parkinson disease}

Mattia Arlotti, Sara Marceglia, Guglielmo Foffani, et al.

Neurology 2018;90;e971-e976 Published Online before print February 14, 2018

DOI 10.1212/WNL.0000000000005121

This information is current as of February 14, 2018

Updated Information \&
Services

Supplementary Material

References

Citations

Subspecialty Collections

Permissions \& Licensing

Reprints including high resolution figures, can be found at: http://n.neurology.org/content/90/11/e971.full

Supplementary material can be found at: http://n.neurology.org/content/suppl/2018/02/14/WNL.0000000000005 121.DC1

This article cites 7 articles, 1 of which you can access for free at: http://n.neurology.org/content/90/11/e971.full\#ref-list-1

This article has been cited by 2 HighWire-hosted articles: http://n.neurology.org/content/90/11/e971.full\#\#otherarticles

This article, along with others on similar topics, appears in the following collection(s):

Basal ganglia

http://n.neurology.org/cgi/collection/basal_ganglia

Motor Control

http://n.neurology.org/cgi/collection/motor_control

Parkinson's disease/Parkinsonism

http://n.neurology.org/cgi/collection/parkinsons_disease_parkinsonism

Information about reproducing this article in parts (figures,tables) or in its entirety can be found online at:

http://www.neurology.org/about/about_the_journal\#permissions

Information about ordering reprints can be found online:

http://n.neurology.org/subscribers/advertise

Neurology ${ }^{\circledR}$ is the official journal of the American Academy of Neurology. Published continuously since 1951, it is now a weekly with 48 issues per year. Copyright Copyright ( 2018 The Author(s). Published by Wolters Kluwer Health, Inc. on behalf of the American Academy of Neurology.. All rights reserved. Print ISSN: 0028-3878. Online ISSN: 1526-632X.

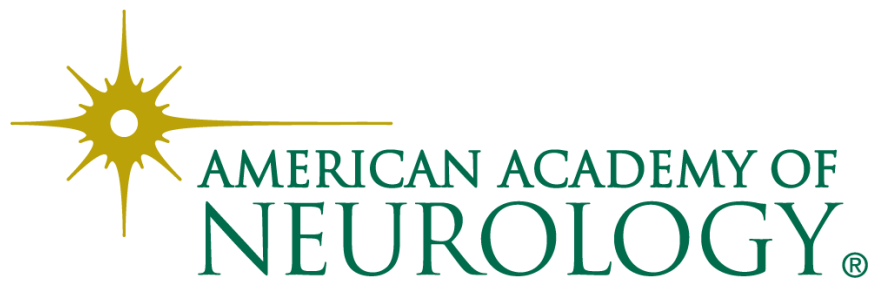

